\title{
Profile of verteporfin and its potential for the treatment of central serous chorioretinopathy
}

This article was published in the following Dove Press journal:

Clinical Ophthalmology

18 September 2013

Number of times this article has been viewed

\author{
Shaheen P Karim \\ Ron A Adelman \\ Department of Ophthalmology \\ and Visual Science, Yale University, \\ New Haven, CT, USA
}

\begin{abstract}
Central serous chorioretinopathy (CSCR) is an idiopathic disorder characterized by serous retinal detachments associated with focal leakage on fluorescein angiography and pigment epithelial detachments. While the majority of cases improve spontaneously over several months, a significant subset of patients advance to a chronic recurrent form of the disease with diffuse pigment epitheliopathy, foveal atrophy, scarring, and permanent visual loss. Photodynamic therapy (PDT) with verteporfin has been extensively studied as a potential therapeutic option for chronic cases. Multiple prospective interventional studies have demonstrated the efficacy of PDT for CSCR with significant functional and anatomic improvements achieved. Refinement of the PDT protocol has subsequently been performed in an effort to minimize adverse effects. Anti-vascular endothelial growth factor (anti-VEGF) agents, such as bevacizumab, have been utilized in the treatment of CSCR. Recent advances in imaging and functional testing have shed further light on possible pathophysiologic mechanisms of disease and post treatment changes induced by PDT. While the body of evidence supports PDT as an efficacious and relatively safe treatment for CSCR, further evaluation of the long-term efficacy and safety of PDT, as well as protocol improvements are required.
\end{abstract}

Keywords: photodynamic therapy, retinopathy, CSC, CSCR

\section{Introduction}

Central serous chorioretinopathy (CSCR) is a well-characterized disorder that consists of serous retinal detachments, usually in the macula, with areas of leakage on fluorescein angiography (Figure 1A and B). Pigment epithelial detachments (PEDs) at the level of the retinal pigment epithelium (RPE) may be present. Early reports of CSCR by von Graefe, ${ }^{1}$ Maumenee, ${ }^{2}$ and Gass $^{3}$ have been followed by extensive investigations of its clinical features, pathophysiologic mechanisms, imaging characteristics, and therapeutic options.

CSCR disproportionately affects healthy younger males in the age range of 20 years -50 years. Symptoms may include metamorphopsia and central scotomas. Risk factors for CSCR include corticosteroid use ${ }^{4}$ and type A personality. ${ }^{5}$ Fortunately, the natural history of most cases consists of a benign course, with spontaneous resolution and improvement in vision in most patients within several months. ${ }^{6}$ A small percentage of patients may experience chronic disease and permanent visual loss with foveal atrophy, scarring, diffuse retinal pigment epitheliopathy, and secondary choroidal neovascularization (CNV). ${ }^{7}$ Extensive efforts have been made to better understand the pathophysiology of CSCR with the goal of developing therapies for these more difficult refractory cases.
Correspondence: Ron A Adelman Yale University, 40 Temple Street, 3rd Floor, New Haven, CT, 065I0, USA Email ron.adelman@yale.edu 


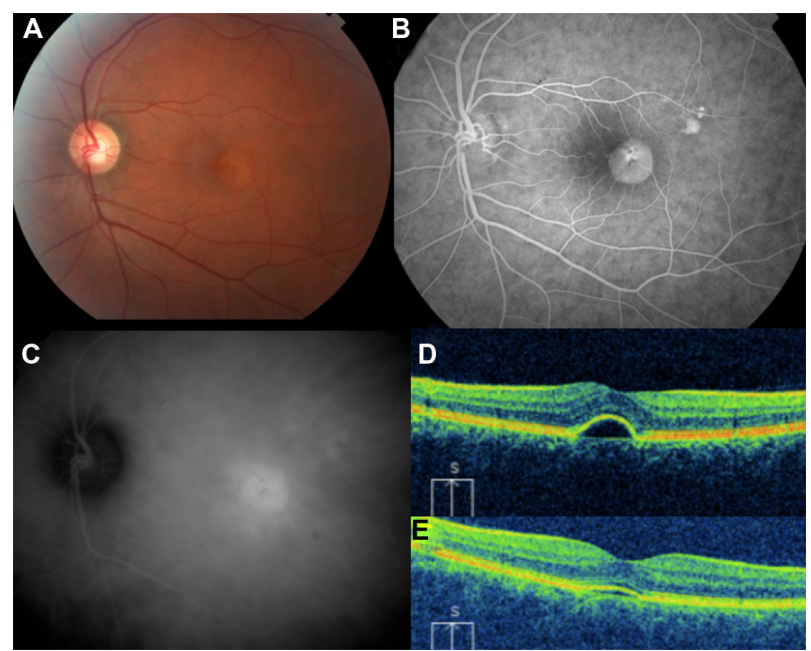

Figure I 4I year old male with history of chronic central serous chorioretinopathy.

Notes: (A) fundus photograph, (B) fluorescein angiogram, (C) ICG angiogram, (D) pre-PDT OCT, and (E) post-PDT OCT showing near resolution 2 months later.

Abbreviations: ICG, indocyanine green; OCT, optical coherence tomography; PDT, photodynamic therapy.

The exact pathophysiologic mechanisms of CSCR are unknown, though hypotheses have been developed with the assistance of fluorescein (FA) and indocyanine green angiography (ICG-A). Typical FA findings include one or two areas of focal juxtafoveal focal leakage at the level of the RPE, with "inkblot" or "smokestack" hyperfluorescence. ${ }^{8}$ ICG-A facilitates evaluation of the choroidal vasculature, with midphase multifocal areas of choroidal hyperfluorescence noted in CSCR patients (Figure 1C). ${ }^{9}$ These areas are postulated to be caused by vascular hyperpermeability. Areas of hyperfluorescence on ICG-A may not only correspond to the clinical detachment and leakage observed with FA, but may also be observed in apparently uninvolved areas and in fellow eyes. Furthermore, clinically silent PEDs may be observed in CSCR patients, with a hyperfluorescent rim accompanied by early central hyperfluorescence and late hyperfluorescence. On the basis of these findings, CSCR is sometimes thought to be a primary disorder of choroidal hyperpermeability from possible inflammation, ischemia, or stasis. ${ }^{10}$ Enhanced depth imaging optical coherence tomography (EDI-OCT) of the choroid has been used to study the choroidal thickness of patients with chronic CSCR. Significantly thicker choroids have been found in patients with chronic CSCR studied by EDI-OCT; a finding supportive of the hypothesis of increased choroidal permeability and hydrostatic pressure. ${ }^{11}$ Physiological discontinuity of the RPE barrier subsequently results in serous retinal detachment. The development of possible treatments for chronic CSCR has focused on targeting these postulated disease mechanisms.

Numerous treatments for chronic CSCR have been investigated, including pharmacologic therapy, laser photocoagulation, and photodynamic therapy (PDT). Vascular endothelial growth factor (VEGF) has been implicated in the modulation of vascular permeability, forming a rational therapeutic approach for chronic CSCR. Indeed, targeted blockage of VEGF by a monoclonal antibody has been attempted in small series with some success, ${ }^{12,13}$ but with other data suggesting anti-VEGF may not be helpful for chronic CSCR. ${ }^{14}$ Larger, long-term studies are required to establish the efficacy of anti-VEGF therapy for chronic CSCR as monotherapy or possible adjuvant therapy. Laser photocoagulation to areas of FA leakage has been attempted, but with limited success and undesired sequelae including photoreceptor damage, scotomas, and disease recurrence. Subthreshold diode laser micropulse photocoagulation has been studied as a refinement of laser therapy for CSCR, showing some promise with early results but requiring further study. ${ }^{15}$

Photodynamic therapy consists of infusion of the photosensitizing medication verteporfin followed by targeted laser activation. Numerous studies have demonstrated its efficacy in improving visual and anatomical outcomes in certain subsets of patients with $\mathrm{CNV}$ secondary to age-related macular degeneration (AMD) ${ }^{16,17}$ In the Treatment of Agerelated macular degeneration with Photodynamic therapy (TAP) Study, ${ }^{16}$ standard PDT protocol consisted of a $6 \mathrm{mg} / \mathrm{m}^{2}$ dosage of verteporfin, fluence of $50 \mathrm{~J} / \mathrm{cm}^{2}, 600 \mathrm{~W} / \mathrm{cm}^{2}$, and laser light exposure for 83 seconds. A proposed mechanism of PDT includes the formation of singlet oxygen and its interaction with the lipids of choroidal endothelial cells, resulting in choroidal endothelial damage, choriocapillaris ischemia, and regression of CNV. ${ }^{18}$ PDT has been investigated in a number of conditions besides CNV secondary to AMD, including choroidal hemangiomas, ${ }^{19} \mathrm{CNV}$ due to pathologic myopia, ${ }^{20}$ and polypoidal choroidal vasculopathy. ${ }^{21}$ Based on the proposed mechanism of increased choroidal vascular permeability in chronic CSCR, targeted PDT may represent a rational therapeutic approach to investigate. While management options for CSCR have been recently reviewed, ${ }^{22,23}$ this paper will further expand on the studies of PDT and its protocol modifications for the treatment of CSCR. Additional areas of interest will be explored, including the potential of molecular modification of the verteporfin molecule, as well as imaging and functional testing after PDT for CSCR. While no standard therapy for chronic CSCR has 
been established on the basis of long-term prospective trials comparing different treatment modalities, a large body of evidence supports the role of PDT.

\section{Early studies of PDT for chronic CSCR}

Yannuzzi et $\mathrm{al}^{24}$ performed a prospective study of 20 eyes in 15 consecutive patients with chronic CSCR, evaluating the effect of ICG-A-guided PDT to improve visual acuity and neurosensory detachments. All patients studied had CSCR for at least 1 year, with an average duration of 9 years. Five eyes previously had focal laser therapy more than 6 months prior to the study. Mean baseline visual acuity was $20 / 275$, ranging from $20 / 40$ to $20 / 800$. Areas of choroidal hyperpermeability identified on ICG angiography were treated with PDT in a manner consistent with the TAP study. Average spot size of the PDT treatment was $4.6 \mathrm{~mm}$ with 12 eyes treated with one spot, and the remaining requiring two spots. All eyes at least maintained their pretreatment vision after PDT, with 12 eyes improving at least two lines on the vision testing chart. Mean visual acuity improved by 0.55 lines $(P=0.05)$ at 6 weeks after treatment. Post-treatment optical coherence tomography (OCT) studies demonstrated complete resolution of the PED in $60 \%$ of eyes with incomplete resolution in the remaining eyes. With no previously known effective therapy for chronic CSCR, this early study of PDT by Yannuzzi et $\mathrm{al}^{24}$ was notable for demonstrating improvements in visual acuity and anatomic outcomes in a set of patients who had longstanding refractory disease.

A similar case series by Cardillo Piccolino et al ${ }^{25}$ also demonstrated improvements in visual acuity and macular detachment by PDT in chronic cases of CSCR. Thirteen consecutive patients with chronic CSCR of at least 6 months duration who had failed focal laser coagulation were studied, with areas of ICG-A hyperpermeability treated with PDT, consistent with the TAP protocol. At the end of the follow up period, 11/16 (69\%) of eyes demonstrated 1-4 lines of visual improvement, with the remainder remaining stable. Complete resolution of subretinal fluid at 1 month after PDT was seen in 12/16 (75\%) eyes, with two eyes showing partial improvement and two eyes showing no change. These functional and anatomic outcomes are slightly better than those published by Yannuzzi, ${ }^{24}$ whose series showed visual improvement in 6/15 (40\%) eyes and a $60 \%$ rate of resolution of subretinal fluid. This difference in outcomes between the two studies may reflect baseline differences in disease severity and duration. Another notable difference between the two studies is that all patients in the Cardillo Piccolino study had prior laser photocoagulation, while only $7 / 15$ eyes in the Yannuzzi study had prior laser photocoagulation.

Taban et $\mathrm{al}^{26}$ published a retrospective study of five eyes in four patients with chronic CSCR who were treated with PDT. Following PDT consistent with the TAP protocol, resolution of the neurosensory detachments was seen in all patients, with significant improvement of vision in 4/5 eyes treated. No recurrences were noted over the average follow up of 10 months.

These early studies provide evidence for the efficacy of PDT for chronic CSCR (Figure 1); however, the changes in the retina and choroid induced by PDT are unknown. Chan et $\mathrm{al}^{27}$ performed quantitative morphometry of the choroidal vasculature following PDT in patients with CSCR. Patients either had persistent CSCR for more than 4 months with focal leakage on FA, or chronic CSCR with diffuse leakage and RPE transmission defects. Following PDT treatment to the areas of congested and dilated choroidal vessels, 4/6 eyes had visual improvement of at least 2 lines, with one patient gaining 1 line and one patient losing 1 line after developing CNV as a complication. Rapid resolution of the focal or diffuse leakage seen on FA was observed in all patients within 3 months, with $83 \%$ resolving at 1 month; a result comparable to Taban et $\mathrm{al}^{26}$ and Cardillo Piccolino et al. ${ }^{25}$ Unlike prior studies, Chan et $\mathrm{al}^{27}$ provided the additional data of quantifying post treatment choroidal changes. Post PDT treatment ICG-A demonstrated narrowing of the previously congested choroidal vessels in all cases, with a mean decrease in diameter of $32 \%$. This study provides additional evidence that PDT may be an effective treatment for chronic CSCR and provides morphologic evidence for the hypothesized post-treatment remodeling of the choroidal vasculature.

While PDT for the treatment of chronic CSCR appears promising in general, clinical responses in early studies were variable, with significant subsets of patients demonstrating incomplete improvement. Inoue et $\mathrm{al}^{28}$ investigated whether the pretreatment appearance of the ICG-A could be associated with treatment efficacy in patients treated with PDT for chronic CSCR. PDT was performed according to the TAP protocol, with the slight modification of a shorter irradiation time of 60-70 seconds instead of 83 seconds. This change resulted in delivered energy levels of $36-42 \mathrm{~mJ} / \mathrm{cm}^{2}$ instead of the usual $50 \mathrm{~mJ} / \mathrm{cm}^{2}$. The investigators studied 32 eyes in 27 patients with chronic CSCR of at least 6 months and with average visual acuity of 0.49 . Pretreatment ICG-A of the studied eyes was categorized as demonstrating intense hyperfluorescence $(72 \%)$, intermediate hyperfluorescence $(19 \%)$, or absent 
hyperfluorescence ( $9 \%$ ) by masked investigators. Overall, in all patients, the rates of subretinal fluid resolution observed at 1 month, 3 months, and 12 months were 59\%, 91\%, and $88 \%$, respectively. In the 23 eyes graded as having intense hyperfluorescence seen on midphase ICG-A, the rates of subretinal fluid resolution at 1 month and 3 months were $70 \%$ and $100 \%$, respectively. In the eyes that demonstrated intermediate hyperfluorescence, the rates of subretinal fluid resolution were $50 \%$ and $100 \%$ at 1 month and 3 months, respectively. There was no resolution of subretinal fluid in any of the three eyes that did not demonstrate hyperfluorescence on baseline ICG-A. The authors conclude that the presence and degree of baseline ICG-A hyperfluorescence may serve as a predictor of efficacy for PDT in chronic CSCR. This data may provide clinical insight regarding which CSCR patients would most likely benefit from PDT. Furthermore, the discrepancies in previously published rates of success for PDT therapy in chronic CSCR may be explained at least in part by this data.

Given that possible mechanisms of PDT include choriocapillaris endothelial damage, hypoperfusion, and ischemia, it is conceivable that the standard full dose PDT initially developed for the treatment of CNV may have adverse effects when used for CSCR. Indeed, reported side effects of PDT treatment for CSCR include choroidal ischemia, secondary choroidal neovascularization, and chronic RPE alterations. CNV was reported in the case series of Chan et al, ${ }^{27}$ as well as in a case report by Colucciello. ${ }^{29}$ In this case report, a patient with a longstanding history of chronic and recurrent CSCR was treated with standard PDT with full resolution of the neurosensory detachment. Ten months following PDT, the patient developed worsening vision with a subretinal macular hemorrhage and evidence of a classic CNV membrane on FA. While CNV development may develop as a complication of CSCR, the temporal relationship in this case suggests the PDT as a possible cause.

Another possible adverse effect of PDT in CSCR is a deleterious alteration of the RPE or neurosensory retina. Cardillo Piccolino et $\mathrm{al}^{25}$ noted RPE changes in $31 \%$ of treated eyes in their series. These changes consisted of retinal atrophy, pigmentary mottling, and lipofuscin-like subretinal deposits. A hypothesized cause was ischemic damage to choroidal vessels induced by PDT. In some cases, it is thought that these adverse pigmentary alterations may have been exacerbated by prior laser photocoagulation therapy. The functional and clinical effects of these post treatment RPE alterations are unclear and require further study. Lai et $\mathrm{al}^{30}$ evaluated pretreatment and post-treatment multifocal electroretinogram (ERG) responses in patients undergoing standard PDT for a number of conditions, including $\mathrm{CNV}$ related to AMD, myopic CNV, idiopathic CNV, and CSCR. While the authors found transient decreases in central and peripheral P1 and N1 responses at 2 weeks that normalized by 1 month, the results are difficult to specifically apply to CSCR as only $2 / 17$ patients included in the study had diagnoses of CSCR.

\section{Modifications of standard PDT protocol for CSCR}

Several investigators have studied modifications to the standard PDT protocol in attempts to minimize potential adverse effects. Possible approaches include decreasing the fluence or decreasing the verteporfin dose. Reibaldi et $\mathrm{al}^{31}$ compared the efficacy of full fluence PDT to half fluence PDT for chronic CSCR in a prospective nonrandomized trial. Outcomes evaluated include change in visual acuity, percentage of eyes with complete resorption of subretinal fluid, change in OCT thickness, and change in choroidal perfusion. Standard PDT dosage was performed according to the TAP study. Half fluence protocol consisted of $6 \mathrm{mg} / \mathrm{m}^{2}$ verteporfin, total light energy of $25 \mathrm{~J} / \mathrm{cm}^{2}$, and a light dose rate of $300 \mathrm{~mW} / \mathrm{cm}^{2}$. This half fluence dose had been previously evaluated by Miller et al ${ }^{32}$ during Phase I and II trials for CNV due to AMD. Nineteen patients received standard fluence PDT and 23 patients received half fluence PDT. Baseline visual acuities were 0.46 and 0.43 in the standard fluence and half fluence groups, respectively. Visual acuity significantly increased in both groups, with mean acuities of 0.27 and 0.28 in the standard and half fluence groups, respectively $(P<0.01)$. However, there was no significant difference between the two groups at any time point. No significant difference was observed in the rate of resorption of subretinal fluid at 12 months between the two groups, with complete resolution seen in $79 \%$ of patients in the full fluence group and $91 \%$ in the half fluence group $(P=0.5)$. Post treatment ICG-A was performed in both groups, with choroidal hypoperfusion graded on a scale developed by Michels. ${ }^{33}$ High-grade hypoperfusion based on this scale was significantly more common in the standard fluence group $(67 \%)$ than in the half fluence group (9\%). One eye treated with standard fluence therapy developed juxtafoveal CNV three months after treatment. These data may provide support that half fluence PDT can achieve comparable, if not possibly superior, outcomes to standard fluence PDT while decreasing the risks of adverse side effects such as CNV and long term choroidal hypoperfusion. 
Shin et $a l^{34}$ also investigated the use of half fluence PDT for chronic CSCR, performing a multicenter retrospective study comparing half fluence to full fluence PDT for CSCR. Half fluence PDT was performed in 34 eyes with history of chronic CSCR while 33 eyes received full fluence PDT. Functional and anatomic outcomes were similar to those previously reported by Reibaldi et al. ${ }^{31}$ The rates of complete resorption of subretinal fluid at 1 month were $94 \%$ and $100 \%$ in the half fluence and full fluence groups, respectively, and were not significantly different between the two groups. Best corrected visual acuity in both groups was significantly improved compared to baseline at all time-points, with final average visual acuities of 0.17 in the half fluence group and 0.21 in the full fluence group. Shin et $\mathrm{al}^{34}$ expanded on prior similar studies by quantifying post PDT choroidal hypoperfusion by quantitative image analysis. Mean gray values for treated and untreated areas were computed from post treatment ICG-A using commercial image analysis software, and a gray value ratio was computed for treated to non-treated areas. With lower grey value ratios suggestive of hypoperfusion, the ratio observed for the full fluence group was significantly lower than in the half fluence group. These data provide further evidence that half fluence PDT for the treatment of chronic CSCR is equally as efficacious as full fluence PDT, and describes a method for post-treatment quantification of choroidal hypoperfusion.

Smretschnig et al ${ }^{35}$ conducted a retrospective review evaluating half fluence PDT for chronic CSCR of at least 6 months duration, reproducing the findings of Shin et $\mathrm{al}^{34}$ and Reibaldi et al. ${ }^{31}$ PDT with $6 \mathrm{mg} / \mathrm{m}^{2}$ verteporfin infusion consisted of $25 \mathrm{~J} / \mathrm{cm}^{2}$ energy delivered with light intensity of $300 \mathrm{~mW} / \mathrm{cm}^{2}$. One year after treatment, best corrected visual acuity (BCVA) improved from an average baseline of 40 letters to 44 letters $(P<0.01)$. Central foveal thickness also significantly decreased from a baseline of 325 microns to 222 microns $(P<0.001)$. OCT performed 1 month after treatment demonstrated resolution of the subretinal fluid in $100 \%$ of eyes. No significant adverse effects such as CNV development or RPE alterations were observed.

Investigators have studied additional modifications to the PDT protocol besides changing the fluence for treating chronic CSCR. Other PDT parameters that could be changed include verteporfin dosage and time to laser activation after infusion. Chan et al ${ }^{36}$ studied decreasing the verteporfin dose of standard PDT protocol to treat chronic CSCR patients, performing a prospective non-randomized interventional case series. Protocol modifications included half dose verteporfin of $3 \mathrm{mg} / \mathrm{m}^{2}$ and laser delivery at 10 minutes following infusion, instead of the usual 15 minutes. Total light energy delivery of $50 \mathrm{~J} / \mathrm{cm}^{2}$ over 83 seconds was maintained from the standard protocol. A total of 48 eyes in 48 patients with evidence of chronic CSCR were treated with the modified PDT protocol. Mean visual acuity significantly increased at 1 year $(P<0.001)$, with $96 \%$ of patients maintaining at least stable vision at 1 year. Central foveal thickness significantly decreased in treated patients, decreasing to 175 microns at 1 year from a mean baseline of 320 microns $(P<0.001)$. At 3 months, $75 \%$ of treated eyes demonstrated complete resolution of subretinal fluid and pigment epithelial detachments, increasing to $90 \%$ of eyes at 1 year. Resolution of ICG-A hyperpermeability following PDT treatment was observed in $79 \%$ of eyes, with partial resolution in ten eyes. With outcomes similar to those published for standard as well as half fluence PDT, these data from Chan et al support the usage of half dose PDT $\left(3 \mathrm{mg} / \mathrm{m}^{2}\right)$ as an effective treatment for chronic CSCR.

Kempton and Adelman ${ }^{37}$ studied combining half dose verteporfin with increasing activation time for the treatment of chronic CSCR by PDT. Activation time was increased to 30 minutes from the standard 15 minutes. The rationale for this modification is that, as fluorescein leakage is slow in CSCR, delayed time to verteporfin activation may allow more verteporfin accumulation at the site of pathologic vessels and thus facilitate more verteporfin binding to pathologic vasculature. Both of the two patients with chronic CSCR studied with half dose verteporfin and delayed activation demonstrated complete resolution of subretinal fluid at 1 month. Delayed activation may be beneficial in chronic CSCR patients undergoing PDT, and warrants further study.

In addition to changing the parameters of standard PDT for CSCR, modification of the verteporfin molecule has also been studied as a way to increase PDT selectivity. Lu et al ${ }^{38}$ modified the verteporfin molecule by conjugating a molecule containing factor VII (fVII). This fVII-verteporfin complex is hypothesized to more selectively target pathologic endothelial cells by binding specifically to tissue factor (TF), a molecule selectively expressed on damaged abnormal endothelial cells. In a rat model of laser induced CNV, treatment with fVIIverteporfin $\left(0.5\right.$ and $\left.1.0 \mathrm{mg} / \mathrm{m}^{2}\right)$ was demonstrated to result in significantly less post-treatment leakage than treatment with standard verteporfin $\left(6 \mathrm{mg} / \mathrm{m}^{2}\right)$. This data suggest that fVII-verteporfin may more specifically target pathologic vasculature than standard verteporfin in rat models of $\mathrm{CNV}$, and that optimization of the verteporfin molecule deserves further investigation for the treatment of human disease. 


\section{Imaging and functional testing of CSCR following PDT}

While multiple studies demonstrated that PDT for chronic CSCR is effective and relatively safe (Table 1), the retinal and choroidal changes induced by therapy are unknown. Recent advances in retinal imaging and functional testing have allowed investigators to better understand posttreatment anatomic and functional alterations induced by PDT for chronic CSCR. In a retrospective comparative series, Maruko et a ${ }^{139}$ used enhanced depth imaging OCT (EDI-OCT) to investigate changes in choroidal thickness in eyes undergoing laser photocoagulation or PDT for chronic CSCR. Laser photocoagulation was performed in patients with areas of focal leakage on FA. Half dose PDT $\left(3 \mathrm{mg} / \mathrm{m}^{2}\right)$ was performed in patients without focal leakage but with persistent serous detachment for more than 6 months. Pre- and post-treatment subfoveal choroidal thickness was measured using the EDI OCT technique. In the patient group receiving laser photocoagulation, the mean subfoveal choroidal thicknesses at baseline, 1 week, and 1 month were 345 microns, 349 microns, and 340 microns, respectively. No significant change between baseline and 1 month was noted. In the patients receiving half dose PDT, mean subfoveal choroidal thickness at baseline was 389 microns, which significantly increased to 462 microns at day $2(P=0.008)$. Subfoveal thickness at 1 week and 1 month then significantly decreased with respect to baseline; 360 microns and 330 microns, respectively $(P=0.001)$. In comparison to the laser photocoagulation group in which subfoveal choroidal thickness did not change, there was an initial post treatment increase in the post PDT group, followed by a decrease at 1 month. These data are consistent with the possibility of increased choroidal exudation immediately after PDT, followed by long-term alterations in choroidal thickness leading to decreased permeability.

Instead of using EDI-OCT to evaluate the choroid, Shinojima et $\mathrm{al}^{40}$ used spectral domain OCT to study morphometric alterations in the retina following half dose PDT for CSCR. A total of 17 eyes of 17 patients with chronic CSCR were treated with $3 \mathrm{mg} / \mathrm{m}^{2} \mathrm{PDT}$, with spectral domain OCT performed before and after treatment. The OCT images were evaluated for presence of inner segment/outer segment (IS/OS) junctions, RPE irregularities, highly reflective substances (HRS), and pigment epithelial detachments. Following PDT, total resolution of the serous retinal detachment was observed in 16/17 eyes by 3 months, with one case recurring at 9 months. At baseline in $4 / 17$ eyes, the IS/OS junction was visualized on OCT. Six months after treatment, the IS/OS line became clearer with visualization of the IS/OS junction in 13/17 eyes. All eyes demonstrated RPE irregularities and highly reflective substances below the RPE prior to PDT, which were unchanged at 1 year after treatment. The exact etiology of the highly reflective substances observed is unknown, but is hypothesized to be from choriocapillaris exudation or occlusion. The authors conclude from this data that, despite rapid resolution of subretinal fluid in the majority of cases following PDT for chronic CSCR, persistent RPE and choroidal alterations can develop that warrant continued close monitoring.

Pryds et al ${ }^{41}$ attempted to shed further light on the clinical significance of hyperautofluorescent subretinal deposits in chronic CSCR noted by Shinojima et $\mathrm{a}^{40}$ and several prior authors. ${ }^{42,43}$ Using microperimetry to assess retinal function, the investigators used PDT in 21 patients with CSCR of at least 4 months duration with serous retinal detachments, autofluorescent subretinal deposits, and late FA leakage.

Table I Photodynamic therapy for chronic central serous chorioretinopathy

\begin{tabular}{|c|c|c|c|c|c|c|c|c|}
\hline Authors & $\begin{array}{l}\text { Number } \\
\text { of eyes }\end{array}$ & $\begin{array}{l}\text { Verteporfin } \\
\text { dose }\left(\mathrm{mg} / \mathrm{m}^{2}\right)\end{array}$ & $\begin{array}{l}\text { PDT fluence } \\
\left(\mathrm{J} / \mathrm{cm}^{2}\right)\end{array}$ & $\begin{array}{l}\text { Resolution } \\
\text { of SRF (\%) }\end{array}$ & $\begin{array}{l}\text { VA improved } \\
\text { (\%) }\end{array}$ & $\begin{array}{l}\text { VA unchanged } \\
\text { (\%) }\end{array}$ & $\begin{array}{l}\text { VA worse } \\
\text { (\%) }\end{array}$ & $\begin{array}{l}\text { Follow up } \\
\text { (months) }\end{array}$ \\
\hline Yannuzzi et $\mathrm{al}^{24}$ & 20 & 6 & 50 & 60 & 30 & 70 & - & $4-12.5$ \\
\hline Taban et $\mathrm{al}^{26}$ & 5 & 6 & 50 & 100 & 80 & 20 & - & $8-12$ \\
\hline Cardillo Piccolino et $\mathrm{al}^{25}$ & 16 & 6 & 50 & 81 & 69 & 31 & - & $6-12$ \\
\hline Chan et $\mathrm{al}^{36}$ & 48 & 3 & 50 & 90 & 96 & & 4 & 12 \\
\hline Shinojima et $\mathrm{al}^{40}$ & 17 & 3 & 50 & 94 & 6 & 6 & 88 & \\
\hline \multirow[t]{2}{*}{ Shin et $\mathrm{al}^{34}$} & 33 & 6 & 25 & 94 & $*$ & - & - & $6-33$ \\
\hline & 34 & 6 & 50 & 100 & $* *$ & - & - & $5-25$ \\
\hline \multirow[t]{2}{*}{ Reibaldi et al $^{31}$} & 23 & 6 & 25 & 91 & $* * *$ & - & - & 12 \\
\hline & 19 & 6 & 50 & 79 & $* * * *$ & - & - & 12 \\
\hline Silva et $\mathrm{al}^{47}$ & 48 & 6 & 50 & 93 & 74 & 17 & 9 & 48 \\
\hline Smretschnig et $\mathrm{al}^{35}$ & 20 & 6 & 25 & 100 & 92 & 8 & - & 1 \\
\hline
\end{tabular}

Notes: *LogMAR change from baseline 0.34 to 0.17 after treatment; **LogMAR change from baseline 0.46 to 0.21 after treatment; *** LogMAR change from baseline 0.46 to 0.16 after treatment; ****LogMAR change from baseline 0.43 to 0.27 after treatment.

Abbreviations: LogMAR, logarithm of the minimum angle of resolution; PDT, photodynamic therapy; SRF, subretinal fluid; VA, visual acuity. 
Autofluorescent subretinal deposits were graded based on size and severity, and microperimetry was used to assess retinal function extending to 6 degrees eccentricity. PDT consisted of full dose verteporfin $\left(6 \mathrm{mg} / \mathrm{m}^{2}\right)$ with light dose intensity of $50 \mathrm{~J} /$ $\mathrm{cm}^{2}$, but with a shortened laser exposure time of 42 seconds. Subgroup analysis was performed, with $11 / 21$ patients presenting with an initial CSCR episode and 10/21 patients presenting with a recurrent episode. All eyes in both groups demonstrated complete resorption of subretinal fluid after 4 months. Microperimetry testing at 4 months demonstrated improvement in retinal sensitivity in both groups. In the initial episode group, mean foveal sensitivity increased from 12.1 $\mathrm{dB}$ at baseline to $15.8 \mathrm{~dB}$ after 4 months $(P=0.005)$. In eyes with recurrent episodes, mean foveal sensitivity increased from $10.5 \mathrm{~dB}$ at baseline to $15.5 \mathrm{~dB}$ at baseline $(P=0.001)$. Both subgroups demonstrated mean deficits in foveal sensitivity compared to unaffected fellow eyes, with mean deficits of 3.1 $(P=0.008)$ and $2.7 \mathrm{~dB}(P=0.028)$ in the initial and recurrent episode groups, respectively. In both groups, post-treatment foveal thickness was significantly lower than foveal thickness in the fellow unaffected eye. There was no association between subretinal deposit grade and foveal thickness after treatment. While PDT may result in resolution of subretinal fluid, this data suggests that patients with CSCR and subretinal deposits demonstrate residual functional deficits and anatomical changes after treatment. Similarly, Ratanasukon et al found photoreceptor disruption in a significant number of CSCR patients following half dose PDT. ${ }^{44}$ While no correlation with visual acuity was found, possible foveal side-effects of PDT therapy should be considered.

Fujita et $\mathrm{al}^{45}$ also studied retinal sensitivity by microperimetry before and after PDT for chronic CSCR. Thirteen eyes in 13 patients with chronic CSCR were enrolled in this prospective interventional case series. Spectral domain optical coherence tomography (SD-OCT) was used to evaluate the external limiting membrane (ELM), IS/OS junction, and cone outer segment tips (COST). Microperimetry with a Goldmann III target evaluating 45 stimulus locations over a $12^{\circ}$ diameter was used to evaluate retinal sensitivity. Following half dose $\left(3 \mathrm{mg} / \mathrm{m}^{2}\right)$ PDT, the serous retinal detachment resolved in 12/13 eyes within 3 months, with eleven eyes resolving at 1 month. All eyes demonstrated an increase in retinal sensitivity of at least $2.5 \mathrm{~dB}$ at 12 months following therapy. The mean retinal sensitivity of $7.2 \mathrm{~dB}$ in the area of serous retinal detachment increased significantly to $15.4 \mathrm{~dB}$ 12 months after half dose PDT. This post-treatment increase in microperimetry sensitivity is similar to that found by Pryds et al. ${ }^{41}$ Compared to previous studies evaluating retinal microperimetry after PDT for CSCR, Fujita et al ${ }^{45}$ provided additional data regarding retinal structural details. Fujita et $\mathrm{al}^{45}$ observed an improvement in the status of the ELM, IS/OS, and COST lines following therapy. While no eyes demonstrated IS/OS and COST lines prior to treatment, 1 year after therapy, 11/13 eyes demonstrated a fragmented or intact IS/OS line and 6/13 eyes demonstrated a fragmented or intact COST line. Subgroup analysis demonstrated that patients with both IS/OS and COST lines had significantly higher retinal sensitivities than eyes without the COST line present, though no significant difference in visual acuity was noted. The data from this study suggest that PDT for chronic CSCR may result in an improvement in retinal sensitivity that is associated with the presence of retinal microstructures such as the IS/OS and COST lines.

In addition to OCT and microperimetry, multifocal ERG (mfERG) has been used to characterize the changes in retinal function following PDT for chronic CSCR. Wu et $\mathrm{al}^{46}$ performed a randomized placebo-controlled trial evaluating mfERG following safety enhanced PDT for acute CSCR of less than 3 months duration. mfERG and OCT were performed at baseline and 1 year after treatment. Half dose PDT was performed in 24 eyes, with a verteporfin dosage of $3 \mathrm{mg} / \mathrm{m}^{2}, 50 \mathrm{~J} / \mathrm{cm}^{2}$, and activation 10 minutes following start of infusion. Ten patients received placebo, consisting of infusion of normal saline instead of verteporfin, followed by laser application in the same manner as the treatment group. Twelve months following treatment, the change in visual acuity in the PDT group was +1.8 lines; a significant increase compared to the +0.1 line gained in the placebo group $(P=0.003)$. Central foveal thickness decreased in the PDT treatment group from a baseline of 486 microns to 160 microns at 12 months after treatment. The placebo group demonstrated a decrease in central foveal thickness from 418 microns to 262 microns at 12 months. With regards to the mfERG testing, the baseline N1 and P1 amplitudes for the PDT and placebo groups demonstrated no significant difference. At 12 months, however, the $\mathrm{P} 1$ responses in the PDT group significantly increased by $56 \%$ and $39 \%$ for the two central rings studied, respectively, compared to $9 \%$ and $6 \%$ for the placebo responses $(P=0.018)$. Based on this additional OCT and $\mathrm{mfERG}$ data, half dose PDT thus appears to improve anatomic and functional outcomes at 1 year after treatment for acute CSCR.

\section{Conclusion}

A mounting body of evidence, including recent long-term data, ${ }^{47}$ demonstrates that PDT is an effective and relatively 
safe treatment for cases of chronic refractory CSCR (Table 1). Modifications of standard PDT protocol have resulted in the decreased likelihood of adverse effects, such as pigment epithelial changes, while demonstrating efficacy in improving visual and anatomic outcomes. Advances in anatomic and functional retinal testing have provided insight into the mechanisms of disease and effects of PDT. Additional prospective studies comparing the efficacy of different PDT protocols for chronic CSCR should be performed. While longer term follow up of safety and efficacy is needed, photodynamic therapy appears to be, on the basis of multiple studies, an effective therapy for CSCR.

\section{Disclosure}

The authors report no conflicts of interest in this work.

\section{References}

1. von Graefe A. Uber centrale recidivierende retinitis [On central recurrent retinitis] Archiv für Ophthalmologie. 1866;12:211-215.

2. Maumenee AE. Macular Diseases: Clinical Manifestations. Trans Am Acad Ophthalmol Otolaryngol. 1965;69:605-613.

3. Gass JDM. Pathogenesis of disciform detachment of the neuroepithelium: II Idiopathic central serous choroidopathy. Am J Ophthalmol. 1967;63:587-615.

4. Carvalho-Recchia CA, Yannuzzi LA, Negrao S, et al. Corticosteroids and central serous chorioretinopathy. Ophthalmology. 2002;109(10): 1834-1837.

5. Yannuzzi LA. Type A behavior and central serous chorioretinopathy. Trans Am Ophthalmol Soc. 1986;84:799-845.

6. Klein ML, Van Buskirk EM, Friedman E, Gragoudas E, Chandra S. Experience with nontreatment of central serous choroidopathy. Arch Ophthalmol. 1974;91(4):247-250.

7. Gass JDM. Stereoscopic Atlas of Macular Diseases: Diagnosis and Treatment, 4th ed. St Louis: Mosby; 1997.

8. Maumenee AE. Doyne Memorial Lecture. Fluorescein angiography in the diagnosis and treatment of lesions of the ocular fundus. Trans Ophthalmol Soc U K. 1969;88:529-556.

9. Guyer DR, Yannuzzi LA, Slakter JS, Sorenson JA, Ho A, Orlock D. Digital indocyanine green videoangiography of central serous chorioretinopathy. Arch Ophthalmol. 1994;112(8):1057-1062.

10. Yannuzzi LA. Central serous chorioretinopathy: a personal perspective. Am J Ophthalmol. 2010;149(3):361-363.

11. Imamura Y, Fujiwara T, Margolis R, Spaide RF. Enhanced depth imaging optical coherence tomography of the choroid in central serous chorioretinopathy. Retina. 2009;29(10):1469-1473.

12. Torres-Soriano ME, Garcia-Aguirre G, Kon-Jara V, et al. A pilot study of intravitreal bevacizumab for the treatment of central serous chorioretinopathy (case reports). Graefes Arch Clin Exp Ophthalmol. 2008;246(9):1235-1239.

13. Lee ST, Adelman RA. The treatment of recurrent central serous chorioretinopathy with intravitreal bevacizumab. J Ocul Pharmacol Ther. 2011;27(6):611-614.

14. Bae SH, Heo JW, Kim C, et al. A randomized pilot study of lowfluence photodynamic therapy versus intravitreal ranibizumab for chronic central serous chorioretinopathy. Am J Ophthalmol. 2011; 152(5):784-792. e2.

15. Koss MJ, Beger I, Koch FH. Subthreshold diode laser micropulse photocoagulation versus intravitreal injections of bevacizumab in the treatment of central serous chorioretinopathy. Eye (Lond). 2012;26(2): $307-314$.
16. No authors listed. Photodynamic therapy of subfoveal choroidal neovascularization in age-related macular degeneration with verteporfin: one-year results of 2 randomized clinical trials - TAP report. Treatment of age-related macular degeneration with photodynamic therapy (TAP) Study Group. Arch Ophthalmol. 1999;117(10):1329-1345.

17. Schmidt-Erfurth U, Miller JW, Sickenberg M, et al. Photodynamic therapy with verteporfin for choroidal neovascularization caused by age-related macular degeneration: results of retreatments in a phase 1 and 2 study. Arch Ophthalmol. 1999;117(9):1177-1187.

18. Schmidt-Erfurth U, Hasan T. Mechanisms of action of photodynamic therapy with verteporfin for the treatment of age-related macular degeneration. Surv Ophthalmol. 2000;45(3):195-214.

19. Schmidt-Erfurth UM, Michels S, Kusserow C, Jurklies B, Augustin AJ. Photodynamic therapy for symptomatic choroidal hemangioma: visual and anatomic results. Ophthalmology. 2002;109(12):2284-2294.

20. Verteporfin in Photodynamic Therapy Study Group. Photodynamic therapy of subfoveal choroidal neovascularization in pathologic myopia with verteporfin. 1-year results of a randomized clinical trial - VIP report no 1. Ophthalmology. 2001;108(5):841-852.

21. Kang HM, Kim YM, Koh HJ. Five-year follow-up results of photodynamic therapy for polypoidal choroidal vasculopathy. Am J Ophthalmol. 2013;155(3):438-447.

22. Quin G, Liew G, Ho IV, Gillies M, Fraser-Bell S. Diagnosis and interventions for central serous chorioretinopathy: review and update. Clin Experiment Ophthalmol. 2012;41(2):187-200.

23. Nicholson B, Noble J, Forooghian F, Meyerle C. Central serous chorioretinopathy: update on pathophysiology and treatment. Surv Ophthalmol. 2013;58(2):103-126.

24. Yannuzzi LA, Slakter JS, Gross NE, et al. Indocyanine green angiography-guided photodynamic therapy for treatment of chronic central serous chorioretinopathy: a pilot study. Retina. 2003; 32 Suppl 1:288-298.

25. Cardillo Piccolino F, Eandi CM, Ventre L, Rigault de la Longrais RC, Grignolo FM. Photodynamic therapy for chronic central serous chorioretinopathy. Retina. 2003;23(6):752-763.

26. Taban M, Boyer DS, Thomas EL. Chronic central serous chorioretinopathy: photodynamic therapy. Am J Ophthalmol. 2004; 137(6):1073-1080.

27. Chan WM, Lam DS, Lai TY, Tam BS, Liu DT, Chan CK. Choroidal vascular remodelling in central serous chorioretinopathy after indocyanine green guided photodynamic therapy with verteporfin: a novel treatment at the primary disease level. Br J Ophthalmol. 2003;87(12):1453-1458.

28. Inoue R, Sawa M, Tsujikawa M, Gomi F. Association between the efficacy of photodynamic therapy and indocyanine green angiography findings for central serous chorioretinopathy. Am J Ophthalmol. 2010;149(3):441-446. e1-e2.

29. Colucciello M. Choroidal neovascularization complicating photodynamic therapy for central serous retinopathy. Retina. 2006;26(2):239-242.

30. Lai TY, Chan WM, Lam DS. Transient reduction in retinal function revealed by multifocal electroretinogram after photodynamic therapy. Am J Ophthalmol. 2004;137(5):826-833.

31. Reibaldi M, Cardascia N, Longo A, et al. Standard-fluence versus low-fluence photodynamic therapy in chronic central serous chorioretinopathy: a nonrandomized clinical trial. Am J Ophthalmol. 2010;149(2):307-315. e2.

32. Miller JW, Schmidt-Erfurth U, Sickenberg M, et al. Photodynamic therapy with verteporfin for choroidal neovascularization caused by age-related macular degeneration: results of a single treatment in a phase 1 and 2 study. Arch Ophthalmol. 1999;117(9):1161-1173.

33. Michels S, Hansmann F, Geitzenauer W, Schmidt-Erfurth U. Influence of treatment parameters on selectivity of verteporfin therapy. Invest Ophthalmol Vis Sci. 2006;47(1):371-376.

34. Shin JY, Woo SJ, Yu HG, Park KH. Comparison of efficacy and safety between half-fluence and full-fluence photodynamic therapy for chronic central serous chorioretinopathy. Retina. 2011;31(1):119-126. 
35. Smretschnig E, Ansari-Shahrezaei S, Hagen S, Glittenberg C, Krebs I, Binder S. Half-fluence photodynamic therapy in chronic central serous chorioretinopathy. Retina. 2013;33(2):316-322.

36. Chan WM, Lai TY, Lai RY, Tang EW, Liu DT, Lam DS. Safety enhanced photodynamic therapy for chronic central serous chorioretinopathy: one-year results of a prospective study. Retina. 2008;28(1): 85-93.

37. Kempton JE, Adelman R. Modified photodynamic therapy for the treatment of central serous chorioretinopathy. Invest Ophthalmol Vis Sci. 2008;49:E-Abstract 3277.

38. Lu F, Hu Z, Sinard J, Garen A, Adelman RA. Factor VIIverteporfin for targeted photodynamic therapy in a rat model of choroidal neovascularization. Invest Ophthalmol Vis Sci. 2009;50(8): 3890-3896.

39. Maruko I, Iida T, Sugano Y, Ojima A, Ogasawara M, Spaide RF. Subfoveal choroidal thickness after treatment of central serous chorioretinopathy. Ophthalmology. 2010;117(9):1792-1799.

40. Shinojima A, Kawamura A, Mori R, Fujita K, Yuzawa M. Detection of morphologic alterations by spectral-domain optical coherence tomography before and after half-dose verteporfin photodynamic therapy in chronic central serous chorioretinopathy. Retina. 2011;31(9): 1912-1920.
41. Pryds A, Larsen M. Foveal function and thickness after verteporfin photodynamic therapy in central serous chorioretinopathy with hyperautofluorescent subretinal deposits. Retina. 2013;33(1):128-135.

42. Spaide RF, Klancnik JM Jr. Fundus autofluorescence and central serous chorioretinopathy. Ophthalmology. 2005;112(5):825-833.

43. von Ruckmann A, Fitzke FW, Fan J, Halfyard A, Bird AC. Abnormalities of fundus autofluorescence in central serous retinopathy. Am J Ophthalmol. 2002;133(6):780-786.

44. Ratanasukon M, Thongthong K, Bhurayanontachai P, Jirarattanasopa P. Photoreceptor disruption in central serous chorioretinopathy treated by half-dose photodynamic therapy. Clin Ophthalmol. 2013;7:87-92.

45. Fujita K, Shinoda K, Imamura Y, et al. Correlation of integrity of cone outer segment tips line with retinal sensitivity after half-dose photodynamic therapy for chronic central serous chorioretinopathy. Am J Ophthalmol. 2012;154(3):579-585.

46. Wu ZH, Lai RY, Yip YW, Chan WM, Lam DS, Lai TY. Improvement in multifocal electroretinography after half-dose verteporfin photodynamic therapy for central serous chorioretinopathy: a randomized placebocontrolled trial. Retina. 2011;31(7):1378-1386.

47. Silva RM, Ruiz-Moreno JM, Gomez-Ulla F, et al. Photodynamic therapy for chronic central serous chorioretinopathy: a 4-Year follow-up study. Retina. 2013;33(2):309-315.
Clinical Ophthalmology

\section{Publish your work in this journal}

Clinical Ophthalmology is an international, peer-reviewed journal covering all subspecialties within ophthalmology. Key topics include: Optometry; Visual science; Pharmacology and drug therapy in eye diseases; Basic Sciences; Primary and Secondary eye care; Patient Safety and Quality of Care Improvements. This journal is indexed on

Submit your manuscript here: http://www.dovepress.com/clinical-ophthalmology-journal

\section{Dovepress}

PubMed Central and CAS, and is the official journal of The Society of Clinical Ophthalmology (SCO). The manuscript management system is completely online and includes a very quick and fair peer-review system, which is all easy to use. Visit http://www.dovepress.com/ testimonials.php to read real quotes from published authors. 